\title{
Chemometric Simultaneous Determination of Atorvastatin and Amlodipine in Bulk and Tablets
}

\author{
Imad Osman Abu Reid*, Malak EIrasheed Mohamed \\ Department of Pharmaceutical Chemistry, Faculty of Pharmacy, National Al Ribat University, Khartoum, Sudan
}

Email address:

iabureid@hotmail.com (I. O. A. Reid)

${ }^{*}$ Corresponding author

To cite this article:

Imad Osman Abu Reid, Malak Elrasheed Mohamed. Chemometric Simultaneous Determination of Atorvastatin and Amlodipine in Bulk and Tablets. International Journal of Pharmacy and Chemistry. Vol. 5, No. 4, 2019, pp. 42-47. doi: 10.11648/j.ijpc.20190504.12

Received: April 5, 2019; Accepted: July 22, 2019; Published: October 9, 2019

\begin{abstract}
A simple, accurate and precise UV-spectrophotometric method based inverse least-squares was developed for the simultaneous determination of atorvastatin and amlodipine in tablet formulation. The absorbance values of the two analytes were linear with the concentration at the wavelengths taken at $5 \mathrm{~nm}$ interval over the range of $230-260 \mathrm{~nm}$. The calibration equations were developed using the absorbance values of nine synthetic mixtures containing different concentrations of two analytes measured at $5 \mathrm{~nm}$ intervalsin the range of $230-260 \mathrm{~nm}$. The developed equations werethen validated by calculating the analytes recovery from the analysis of a set of another five synthetic mixtures, the mean $\%$ recoveries were $100.02 \%$ and $100.06 \%$ with the corresponding\% RSD of \pm 0.36 and \pm 0.51 for atorvastatin and amlodipine, respectively. The calibration equations obtained were then used to obtain the concentration of each analyte in commercial samples. The mean $\%$ recoveries were $100.43 \%$ and $100.28 \%$ with the corresponding\% RSD of \pm 0.78 and \pm 0.85 for atorvastatin and amlodipine, respectively. The validity of the proposed method was confirmed through the statistical comparison of the obtained results with those obtained by a reference method utilizing high performance liquid chromatography for the determination of the two actives, the calculated $\mathrm{t}$-values at $(\mathrm{P}=0.05, \mathrm{n}=6)$ were 1.47 and 0.73 compared to the tabulated value of 2.23 .
\end{abstract}

Keywords: Chemometric, Determination, Atorvastatin, Amlodipine

\section{Introduction}

Amlodipine besylate (AML) chemically is 3-ethyl-5methyl (4RS0-2-[(2-aminoethoxy)methyl]-4chlorophenyl)-6-methyl-1,4-dihydropyridine-3,5dicarboxylate benzenesulphonate. It is used is used in the management of hypertension and angina pectoris, it exerts its effect by blocking calcium ions transmembrane influx into vascular smooth muscles and cardiac smooth muscles [1].

Chemically Atorvastatin calcium (AVS) is [R- (R, R*)]-2(4-fluorophenyl)- $\beta$ - $\delta$-dihydroxy- 5 (1- methylethyl)-3phenyl-4 [ (phenylamino) carbonyl] - 1H-pyrrole-1-heptanoic acid, calcium salt $(2: 1)$ trihydrate. It is a lipid lowering agent which inhibits the conversion of HMG-CoA to mevalonic acid a rate limiting step in hepatic cholesterol production. It is also used for primary and secondary prophylaxis of cardiovascular events [2].

The combination of AML and AVS as antihypertensive and lipid-lowering medications clinically used to reduce the risk of coronary artery disease, stroke and death in patients with cardiovascular risk factors [3].

Determination of multicomponent pharmaceutical dosage forms presents special challenge to the analytical chemists, as in most cases the spectral band(s) of one component overlaps with that of the other(s). Different approaches involving mathematical manipulation of the spectral data have been developed to resolve the overlapping bands. The approach followed principally varies with the extent of overlapping and the number of components involved [4-5].

The simultaneous determination of amlodipine besylate and atorvastatin calcium combination as tablets dosage form is not yet official in any compendia, however literature survey revealed that there are several reported methods; using analytical techniques such as chromatography, spectrophotometry, spectrofluorimetry, electrochemistry and chemometrics for the simultaneous determination of AML and AVS in binary mixtures [6-30].

The overlain absorbance spectra of AML and AVS (Figure 
1) showed considerable overlapping in the range of $230-290$ $\mathrm{nm}$, hence application of the classical spectrophtometric techniques for the determination of their concentration in combined dosage forms is not possible. It is of interest to apply the inverse least-squares method (ILS) using UVspectrophotometry for the simultaneous determination of
AML and AVS combination in tablet dosage form. The success of the attempt is rewarding as it means that a simple, inexpensive, and reliable UV-spectrophotometric method can be used in place of expensive techniques based on separation for their determination.

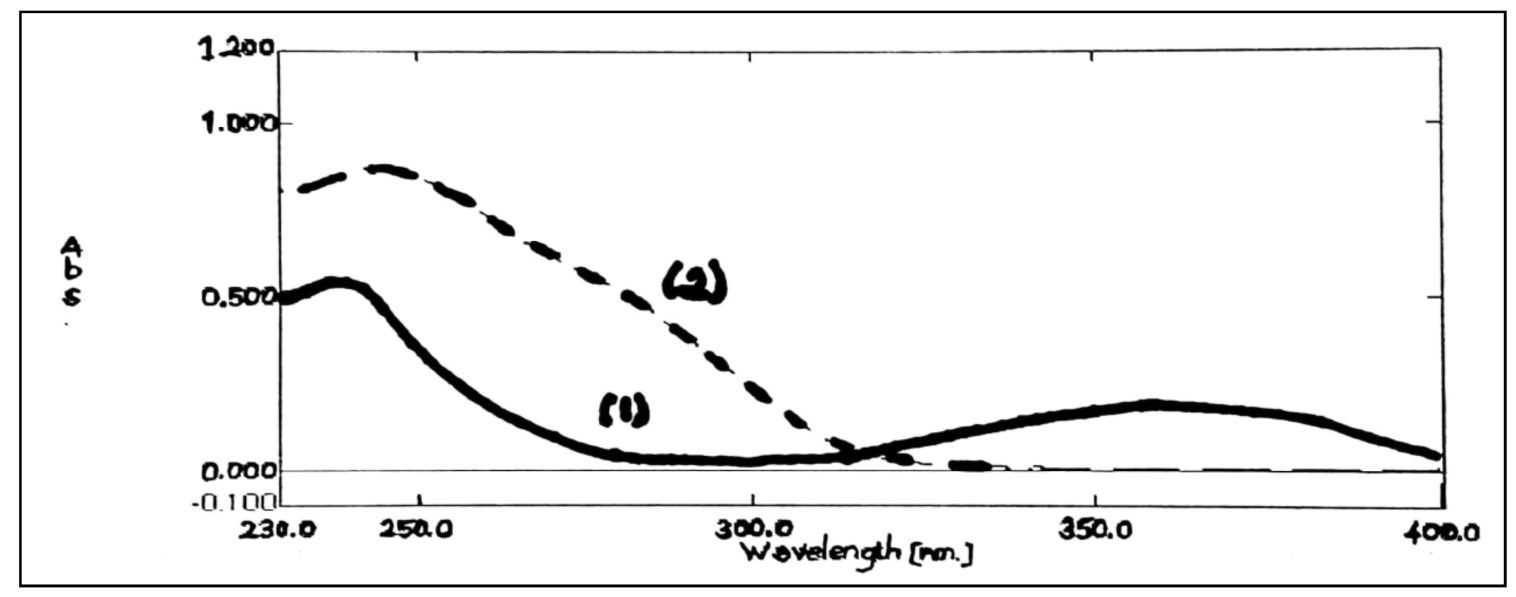

Figure 1. Overlay absorbance spectra of (1) amlodipine besylate (7.5 $\mu \mathrm{g} / \mathrm{ml})$ and (2) atorvastatin calcium (20 $\mu \mathrm{g} / \mathrm{ml}) \mathrm{in} 50 \%$ aqueous methanol.

\section{Theoretical Background}

Inverse least-squares (ILS), sometimes also known as Pmatrix calibration, because, originally, it involved the application of multiple linear regression (MLR) to the inverse expression of the Beer-Lambert Law ofspectroscopy [31]:

$$
\mathrm{C}=\mathrm{PA}
$$

where: concentration $\mathrm{C}$, is a function of absorbance, A. This is the inverse of classical method where (A) is afunction of (C).

For the binary mixture, two concentration variables are involved, namely $\mathrm{C} 1$ and $\mathrm{C} 2$. The application of inverse least-squarestechnique generally executed in the following order:

Computation of the Calibration

Several standard solutions are prepared containing known varying concentrations of the pure the components under analysis (two components in this work). The absorbance of each of these solutions is measured at $\lambda 1, \lambda 2, \lambda 3, \lambda 4, \lambda 5$ etc... to give correspondingly A1, A2, A3, A4, A5 etc... (The number of wavelengths selected should be greater than the number of components).

The absorbance data obtained are processed by suitable software program such as Minitab 16 to give two calibration equations. The calibration equations (Eq. 2 and Eq. 3) are worked out by multiple linear regressions based on the principles of least-squares.

$$
\begin{aligned}
& C_{1}=k_{1}+\alpha_{1} A_{1}+\alpha_{2} A_{2}+\alpha_{3} A_{3}+\alpha_{4} A_{4}+\alpha_{5} A_{5} \text { etc } \\
& C_{2}=k_{2}+\beta_{1} A_{1}+\beta_{2} A_{2}+\beta_{3} A_{3}+\beta_{4} A_{4}+\beta_{5} A_{5} \text { etc }
\end{aligned}
$$

$\mathrm{C} 1$ and $\mathrm{C} 2$ are concentrations of the components of the binary mixture, $k_{1}$ and $k_{2}$ are constants, $\alpha_{i}$ and $\beta_{i}$ are coefficients. The constants and coefficients are worked outby the software program used.

\section{Validation of the calibration equation}

Another set of mixtures with known concentration of the components under analysis is prepared in the same manner as in (I) above andthe absorbance values are measured at the same wavelengths used in the calibration process. The measured absorbance values are then substituted in the calibration equations ( 2 and 3 ) togive $\mathrm{C}_{1}$ and $\mathrm{C}_{2}$.

The validity of the calibration equations is confirmed by obtaining by good recovery and low percent relative standard deviation values $<2 \%$ for the components assayed.

Samples analysis

Similarly, solutions of the samples are treated by measuring the absorbances, $A_{1}, A_{2}, A_{3}, A_{4}, A_{5}$ etc...at thecorresponding wavelengths $\lambda_{1}, \lambda_{2}, \lambda_{3}, \lambda_{4}, \lambda_{5}$ etc... The measured absorbance values of the samples arethen substituted in the calibration equations (2 and 3 ) togive $C_{1}$ and $\mathrm{C}_{2}$.

\section{Materials and Methods}

\subsection{Instrument}

A double beam UV/Vis spectrophotometer, Shimadzu UV1800, was employed with a matching pair of $1 \mathrm{~cm}$ quartz cells for all analytical work.

\subsection{Chemicals and Reagents}

Amlodipine besylate and atorvastatin calcium working standards were provided as a gift by Amipaharma Pharmaceuticals Industry-Sudan. Methanol of analytical grade and double distilled water were used throughout the analysis. Methanol $50 \% \mathrm{v} / \mathrm{v}$ in water was used as a diluent.

\subsection{Commercial Formulation}

Lorvast plus ${ }^{\circledR}$ tablets manufactured by Tabuk 
Pharmaceuticals, Sudan, labeled to contain $20 \mathrm{mg}$ of Atorvastatin as calcium and $5 \mathrm{mg}$ of Amlodipine as besylate were purchased from the local market.

\subsection{Preparation of Stock Standard Solutions}

Usingthe diluents to give final concentration of amlodipine and atorvastatin $54 \mu \mathrm{g} / \mathrm{ml}$ andAccurately weighed $7.5 \mathrm{mg}$ of amlodipine besylate (equivalent to $5.4 \mathrm{mg}$ amlodipine) and $35 \mathrm{mg}$ atorovastatin calcium (equivalent to $32.3 \mathrm{mg}$ atorvastatin) working standards were transferred into two separate $100 \mathrm{ml}$ volumetric flask, dissolved using methanol and completed to mark $323.3 \mu \mathrm{g} / \mathrm{ml}$ respectively.

\subsection{Calibration Curves}

Aliquot volumes $(1-5 \mathrm{ml})$ of each stock solution were transferred into two separate sets of five different $50 \mathrm{ml}$ volumetric flasks and made to mark with the diluents, to give concentration of amlodipine and atorvastatin 1.08-5.4 $\mu \mathrm{g} / \mathrm{ml}$ and $6.5-32.3 \mu \mathrm{g} / \mathrm{ml}$ respectively. The absorbances of the calibration sets weremeasured over the range230-260 nmin 5 $\mathrm{nm}$ intervals andthelinear regression parameters were obtained for the absorbance values against their corresponding concentrations.

\subsection{Preparation of the Calibration Mixtures}

Nine laboratory prepared mixtures containing different concentrations of amlodipine and atorvastatin; covering the expected analytes concentration in the sample were prepared by mixing different volumes from the stock standard solutions of the two analytes in nine separate $50 \mathrm{ml}$ volumetric flasks, the volumes of the flaks were made to markwith the diluent.

\subsection{Preparation of Validation Mixtures}

Five laboratory prepared mixtures containing different concentrations of amlodipine and atorvastatin were prepared by mixing different volumes of the stock standard solutions in five separate $50 \mathrm{ml}$ volumetric flasks, the volumes of the flaks were made to markwith the diluent. The absorbance of the mixtures was measured over the range230-260 nm in5 $\mathrm{nm}$ intervals and used to determine their corresponding concentrations by substituting the measured values in the calibration equations.

\subsection{Sample Preparation}

Twenty tablets were weighed and finely powdered in a mortar, and the average weight of tablet was calculated. An amount ofpowder equivalent to one tablet was accurately weighed, transferred into a100ml volumetric flask and dissolved in methanol with sonication for 15 minutes and completed to the mark with the dilutent. The solution was then filtered using $0.45 \mu \mathrm{m}$ nylon filter; $4 \mathrm{ml}$ of the filtrate were diluted to $50 \mathrm{ml}$ with the dilutent. The absorbance of the sample solution was measured over the range230-260 nm in5 nm intervals and used to calculate the concentration of each analyte by direct substitution in the calibration equations.

\section{Results and Discussion}

Linear regression analysis of the absorbance values of amlodipine and atorvastatin over the range of $230-260 \mathrm{~nm}$ in5 $\mathrm{nm}$ intervals against their corresponding concentrations were performed. The two analytes showed good correlation between the absorbance and concentration $\left(\mathrm{r}^{2}>0.99\right)$. The regression analysis data of the two analytes are shown in Table 1.

Table 1. Analytes regression analysis data.

\begin{tabular}{lll}
\hline \multirow{2}{*}{ Wavelength (nm) } & Regression analysis equation $(\mathbf{y}=\mathbf{b x}+\mathbf{a})$ & \\
\cline { 2 - 3 } & Amlodipine & Atorvastatin \\
\hline 230 & $\mathrm{y}=0.0617+0.0190\left(\mathrm{r}^{2}=0.9982\right)$ & $\mathrm{y}=0.0351 \mathrm{x}+0.0115\left(\mathrm{r}^{2}=0.9984\right)$ \\
235 & $\mathrm{y}=0.068+0.0309\left(\mathrm{r}^{2}=0.9982\right)$ & $\mathrm{y}=0.0388-0.0066\left(\mathrm{r}^{2}=0.9996\right)$ \\
240 & $\mathrm{y}=0.0673 \mathrm{x}+0.0047\left(\mathrm{r}^{2}=0.9996\right)$ & $\mathrm{y}=0.0423 \mathrm{x}-0.0047\left(\mathrm{r}^{2}=0.9997\right)$ \\
245 & $\mathrm{y}=0.0574 \mathrm{x}-0.0051\left(\mathrm{r}^{2}=0.9999\right)$ & $\mathrm{y}=0.0437 \mathrm{x}+0.0137\left(\mathrm{r}^{2}=0.9979\right)$ \\
250 & $\mathrm{y}=0.0413 \mathrm{x}-0.0089\left(\mathrm{r}^{2}=0.9982\right)$ & $\mathrm{y}=0.044 \mathrm{x}-0.0131\left(\mathrm{r}^{2}=0.9988\right)$ \\
255 & $\mathrm{y}=0.02801 \mathrm{x}+0.0049\left(\mathrm{r}^{2}=0.9978\right)$ & $\mathrm{y}=0.0422 \mathrm{x}-0.009\left(\mathrm{r}^{2}=0.9997\right)$ \\
260 & $\mathrm{y}=0.0212 \mathrm{x}-0.0048\left(\mathrm{r}^{2}=0.9979\right)$ & $\mathrm{y}=0.377 \mathrm{x}-0.0009\left(\mathrm{r}^{2}=0.9999\right)$ \\
\hline
\end{tabular}

\subsection{Computation of Calibration Equations}

Table 2 displays the absorbance values of the nine synthetic mixtures used for computation of the constants and the coefficient of the calibration equations using Minitab 16 software.

The following calibration equations were obtained:

$$
\begin{gathered}
\mathrm{C} 1=-1.38+28.57 \mathrm{~A} 230-0.0003 \mathrm{~A} 235-30.38 \mathrm{~A} 240+23.78 \mathrm{~A} 245-78.17 \mathrm{~A} 250+83.41 \mathrm{~A} 255+3.73 \mathrm{~A} 260 \\
\mathrm{R} 2=1.00 \\
\mathrm{C} 2=0.049-8.16 \mathrm{~A} 230-0.0005 \mathrm{~A} 235+23.68 \mathrm{~A} 240+1.23 \mathrm{~A} 245+35.12 \mathrm{~A} 250-57.75 \mathrm{~A} 255+1.37 \mathrm{~A} 260
\end{gathered}
$$

$$
\mathrm{R} 2=1.00
$$


where

$\mathrm{A}_{\mathrm{i}}$ are the absorbance values of the binary mixtures at the selected wavelengths, $\mathrm{C} 1$ and $\mathrm{C} 2$ stand for the concentrations of atorvastatin and amlodipine, respectively. The coefficient of determination, R2, indicated excellent linearity.

Table 2. Absorbance data of the synthetic mixtures.

\begin{tabular}{|c|c|c|c|c|c|c|c|c|c|}
\hline \multirow{2}{*}{ Mix. No. } & \multicolumn{2}{|c|}{ Concentration } & \multicolumn{7}{|c|}{ Absorbance } \\
\hline & AVS & AML & $\lambda 230$ & $\lambda 235$ & $\lambda 240$ & $\lambda 245$ & $\lambda 250$ & $\lambda 255$ & $\lambda 260$ \\
\hline 1 & 19.40 & 3.24 & 0.990 & 1.088 & 1.162 & 1.146 & 1.083 & 0.984 & 0.822 \\
\hline 2 & 12.93 & 4.33 & 0.770 & 0.905 & 0.929 & 0.894 & 0.813 & 0.726 & 0.611 \\
\hline 3 & 25.87 & 4.33 & 1.229 & 1.478 & 1.546 & 1.562 & 1.451 & 1.33 & 1.175 \\
\hline 4 & 25.87 & 3.24 & 1.226 & 1.389 & 1.473 & 1.505 & 1.427 & 1.302 & 1.149 \\
\hline 5 & 19.40 & 4.33 & 1.035 & 1.185 & 1.22 & 1.201 & 1.114 & 1.003 & 0.847 \\
\hline 6 & 25.87 & 2.16 & 1.172 & 1.327 & 1.406 & 1.425 & 1.372 & 1.266 & 1.135 \\
\hline 8 & 12.93 & 3.24 & 0.754 & 822 & 0.858 & 0.871 & 0.794 & 0.698 & 0.600 \\
\hline 9 & 19.40 & 2.16 & 0.933 & 1.077 & 1.055 & 1.102 & 1.057 & 0.951 & 0.853 \\
\hline
\end{tabular}

\subsection{Checking the Validity of the Calibration Equations}

The recovery and percent relative standard deviation (RSD\%) and data obtained by application of the mixtures for AVS and AML by ILS have been summarized in Table 3. The statistical parameters of recovery percentagewere used to evaluate the percent relative standard deviation for theproposed models.

As shown in Table 3, values of RSD\% and recovery for both compounds by ILS method indicate that the proposed method is suitable for successful determination of the two analytes in combination without any prior separation.

Table 3. Model validation data (recovery from synthetic mixtures).

\begin{tabular}{|c|c|c|c|c|c|}
\hline Atorvastatin $(\mu \mathrm{g} / \mathrm{ml})$ & AVS recovered $(\mu \mathrm{g} / \mathrm{ml})$ & \% Recovery & Amlodipine ( $\mu \mathrm{g} / \mathrm{ml})$ & AML recovered $(\mu \mathrm{g} / \mathrm{ml})$ & \% Recovery \\
\hline 19.33 & 19.26 & 99.66 & 3.23 & 3.22 & 99.58 \\
\hline 12.94 & 12.94 & 100.02 & 4.33 & 4.33 & 100.01 \\
\hline 25.80 & 25.73 & 99.73 & 4.31 & 4.30 & 99.66 \\
\hline 26.02 & 26.17 & 100.58 & 3.28 & 3.31 & 100.97 \\
\hline 19.42 & 19.44 & 100.11 & 4.33 & 4.33 & 100.10 \\
\hline Mean \% recovery & & 100.02 & Mean $\%$ recovery & & 100.06 \\
\hline RSD (\%) & & 0.36 & $\operatorname{RSD}(\%)$ & & 0.51 \\
\hline
\end{tabular}

\subsection{Analysis of Commercial Sample}

Table 4 exhibits the\% recoveries obtained by applying the developed chemometric method to the simultaneous determination of atorvastatin and amlodipine in tablet dosage form. The mean $\%$ recoveries were $100.43 \%$ and $100.28 \%$ with the corresponding $\%$ RSD of \pm 0.78 and \pm 0.85 for atorvastatin and amlodipine, respectively.

Table 4. Values of \% recoveries of atorvastatin and amlodipine in tablet formulation.

\begin{tabular}{llllll}
\hline Atorvastatin $(\boldsymbol{\mu g} / \mathbf{m l})$ & AVS recovered $(\boldsymbol{\mu g} / \mathbf{m l})$ & \% Recovery & Amlodipine $(\boldsymbol{\mu g} / \mathbf{m l})$ & AMLrecovered $(\boldsymbol{\mu g} / \mathbf{m l})$ & \% Recovery \\
\hline 14.86 & 14.93 & 100.51 & 2.88 & 2.87 & 99.83 \\
14.84 & 14.90 & 100.40 & 2.87 & 2.86 & 99.59 \\
14.70 & 14.61 & 99.44 & 2.90 & 2.91 & 100.48 \\
15.02 & 15.26 & 101.62 & 2.90 & 2.92 & 100.59 \\
14.74 & 14.71 & 99.75 & 2.93 & 2.99 & 101.75 \\
14.90 & 15.03 & 100.83 & 2.87 & 2.85 & 99.45 \\
Mean\% recovery & & 100.43 & Mean\% recovery & & 100.28 \\
RSD\% & 0.78 & RSD\% & & 0.85 \\
\hline
\end{tabular}

The validity of the method was further assessed by statistically comparing the results obtained with those of a reported high performance liquid chromatographic method [30]. No significant difference between the two methods observed at $(\mathrm{P}=0.05 ; \mathrm{n}=6)$, accordingly the developed method can be considered as accurate and precise as the reported liquid chromatographic method (Table 5).

Table 5. Results of the proposed method compared to the reference method [30].

\begin{tabular}{llll}
\hline & & \% content \pm sd & t calculated (t - tabulated) \\
\hline \multirow{2}{*}{ Proposed method } & Amlodipine & $100.28(0.86)$ & $1.47(2.23)$ \\
& Atorvastatin & $100.43(0.57)$ & $0.73(2.23)$ \\
\multirow{2}{*}{ Reference method } & Amlodipine & $100.96(0.73)$ & $100.13(0.63)$ \\
& Atorvastatin & 1 & \\
\hline
\end{tabular}




\section{Conclusions}

A simple, accurate and precise UV-spectrophotometric method based on chemometrics was developed for the simultaneousdetermination of atorvastatin and amlodipine intablet formulation without prior separation. The cost effectiveness in term of time and moneyrenders the method as suitablealternative to otherexpensive techniques e.g. chromatographic methods for theanalysis of binary mixtures of compounds with overlappedspectra in laboratories and countries where such sophisticated equipments are not affordable. The accuracy and simplicity of the method suggest it suitability in caseswhere quick results are demanded e.g. as an in-process analysis procedure during blend analysis in industrial setups.

\section{Conflict of Interest}

The authors do not have any possible conflicts of interest.

\section{References}

[1] Cardiovascular Drugs. In Martindale, the Complete Drug Reference, Edited by Sweetman SC. Pharmaceutical Press, London. 2009; 36th Edn: 1214.

[2] Cardiovascular Drugs. In Martindale, the complete drug reference, Edited bySweetman SC. Pharmaceutical Press, London. 2009; 36th Edn: 1218-1219.

[3] McKeage $\mathrm{K}$ and Siddiqui MA. Amlodipine/Atorvastatin Fixed-Dose Combination: A Review of its Use in the Prevention of Cardiovascular Disease and in the Treatment of Hypertension and Dyslipidemia. Am. J. Cardiovasc. Drugs 2008; 8: 51-67.

[4] Kamal AH, El-Malla SF and Hammad SF. A review on UV spectrophotometric methods for simultaneous multicomponent analysis. Euro. J. Pharm. Med. Res. 2016; 3 (2): 348-360.

[5] Lotfy HMand Saleh SS. Recent development in ultraviolet spectrophotometry through the last decade (2006-2016): a review. Int. J. Pharm. Pharmac. Sci. 2016; 8 (10): 40-56

[6] Abdallah OM and Badawey AM. Derivative- Ratio Spectrophotometric, Chemometric and HPLC Validated methods for Simultaneous Determination of Amlodipine and Atorvastatin in Combined Dosage Form. Int. J. Ind. Chem. 2011; 2 (2): 78-85.

[7] Darwish HW, Hassan SA and Salem MY, B AEl-Zeand. Development and Validationof H-point Standard Addition Method Appliedforthe Analysisof BinaryMixtrue ofAmlodipineand Atorvastatin. Int. J. Pharm. Bio. Sci. 2013; 4 (2): 230- 243 .

[8] Sarrafi AHM, KonozE and Ghiyasvand M. Simultaneous Detemination of Atorvastatin Calcium and Amlodipine Besylate by Spectrophotometry and Multivariate CalibrationMethods in Pharmaceutical Formulations. EJournal of Chemistry2011; 8 (4): 1670-1679.

[9] Silvia I, Klára K and Daniela-Luci M. Simultaneous Determination of Atorvastatin and Amlodipine in Industrial
Tablets byApparent Content Curve and HPLC Methods. Acta Medica Marisiensis 2013; 59 (1): 44-48.

[10] Bernard S, Mathew M, Senthilkumar KL and Girija KN. Simultaneous Estimation of Atorvastatin Calcium and Amlodipine besylate by UV Spectrophotometric method using hydrotropic solubilization. Hygeia. J. D. Med. 2013; 5 (1): 105-112.

[11] Sahu R and Patel BV. Simultaneous Spectrophotometric Determination of Amlodipine besylate and Atorvastatin calcium in Binary Mixture. Indian J. Pharm. Sci. 2007; 69 (1): 110-111.

[12] Hmadi FA and Am GR. Spectrophotometric Determination of Atorvastatin and Amlodipine by H-point Stndard Addition Methodwith Simultaneous Addition of Both Analalytes in Nonaqueous Solution. Collect. Czech. Chem. Commun. 2011; 76 (3): 193-205.

[13] Tehrani MB, Shoorvazi M and Souri E. Stability Indicating Derivative Spectrophotometric Method for Simultaneous Determination of Amlodipine and Atorvastatin in Pharmaceutical Dosage Forms. Research J. Pharm. Biol. Chem. Sci. 2014; 5 (3): 26-34.

[14] Darwish HW, Hassan SA, Salem MY and El-Zeany BA. Three different methods for determination of binary mixture of Amlodipine and Atorvastatin using dual wavelength spectrophotometry. Spectrochimica Acta Part A: Molecular and Biomolecular Spectroscopy 2013; 104: 70-76.

[15] Sharma K, Sharma Yand Sharma P. Validated Method Developmentfor Estimation of Atorvastatin and Amlodipine in Solid Dosage Regimens. Int. J. Res. Dev. Pharm. L. Sci. 2013; 2 (2): 344-348.

[16] Jena A, Madhu M and Latha S. Analytical Method Developmentand Validation of Simultaneous Determination of Atorvastatin calcium and Amlodipine besylate in Tablet Dosage Form by RP-HPLC. IJPSR, 2010; 1 (11): 100-106.

[17] Hassan SA, Elzanaly ES, Salem MY and EL-Zeany BA. Development and validation of HPLC and CE methods for simultaneous determination of amlodipine and atorvastatinin the presence of their acidic degradation products in tablets. Acta Pharm. 2016; 66: 479-490.

[18] Rao NM and Sankar DG. Development and Validation of HPTLC Method for theSimultaneous Estimation of Amlodipine Besylate and Atorvastatin Calcium in Combined Dosage Form. Eurasian Journal of Analytical Chemistry 2016; 11 (3): 155-168.

[19] Hafez HM, Elshanawany AA, Abdelaziz LM and Mohram MS. Development of a Stability-Indicating HPLC Method for Simultaneous Determination of Amlodipine Besylate and Atorvastatin Calcium in Tablets. Austin J Anal Pharm Chem. 2014; 1 (6): 1- 11.

[20] Mathew M, Bernard S, Senthil Kumar KL and GirijaKN. Development and validation of a high performance thin layer chromatographic method for the simultaneous estimation of Atorvastatin calcium and Amlodipine besylate as the bulk drugs and in the tablet dosage form. Asian J. Pharm. Hea. Sci. 2014; 4 (2): 986-991.

[21] Eranki RJV, Intil G and JayaramanV. New Stability Indicating Method for Quantification of Impurities in Amlodipine and Atorvastatin calcium Tablets by Validated HPLC. Int. J. Pharm. Res. Schol. 2013; 2: 458-471. 
[22] ToanPD, MinhPPAand Trucly DT. Novel HPLC-UV Method Using Volatile Buffer for Simultaneous Determination of Amlodipine Besylateand Atorvastatin Calcium. Int. J. of Pharm. Pharmac. Sci. 2016; 8 (7): 365-368.

[23] Vidyadhara S, Sasidhar RLC, Satyaprasanna P, Aruna KC and Kumari AG. RP-HPLC method development for the simultaneous estimation ofatorvastatin and amlodipine besylate in bulk and pharmaceutical dosage forms. Der Pharmacia Sinica 2015; 6 (10): 1-7.

[24] Moussa BA, El-Zaher A, Mahrouse MA and AhmedMS simultaneous Determination of Amlodipine Besylate andAtorvastatin Calcium in Binary Mixture by Spectrofluorimetry and HPLC coupled with Fluorescence Detection. Analytical Chemistry Insights 2013; 8: 107-115.

[25] IbrahimN, RizkM, IbrahimA, Tawakkol S and ALI I. Simultaneous Determination of Amlodipine Besylateand Atorvasrtatin Caciumby Using Spectrophotometric Method with Multivariate Calibration and HPLC Method Implementing "Design of Experiment". Int J Pharm Pharm Sci. 2014; 6 (1): 419-425.

[26] Ahmed M, Alshabrawy A and Nageh A. UPLC-MS/MS Method for Kinetic Studies and Simultaneous Determination of Amlodipine and Atorvastatin in Bulk, and Their Combined Dosage Form. Anal Chem Ind J. 2016; 16 (14): 102-115.
[27] Dogan-Topal B, Bozal B, Demircigil BT, Uslu B and Ozkan SA. Electroanalytical Studies and Simultaneous Determination of Amlodipine Besylate and Atorvastatine Calcium in Binary Mixtures Using First Derivative of the Ratio-Voltammetric Methods. Electroanalysis 2009; 21 (22): 2427-2439.

[28] Rebech I, Salamanca-Neto CAR, Scremin J and Sartori ER. Simultaneous Voltammetric Determination of Amlodipine andAtorvastatin on Anodically Pretreated Boron-Doped Diamond Electrode Orbital: Electron. J. Chem. 2017; 9 (4): 225-233.

[29] MirciaE, Balaci T, HancuG, IonVand Carje A. Simultaneous Determination of Amlodipine and Atorvastatin by Capillary Electrophoresis from Fixed Pharmaceutical Formulations. Farmacia 2016; 64 (3): 398-402.

[30] Al- Rasoul B, Elsamani T. Validated RP- - HPLC Method for the Simultaneous Determination of Amlodipine Besylate and Atorvastatin Calcium in Bulk and Pharmaceutical Formulation. International Journal of Research in Pharmacy and Chemistry. 2016; 6 (4): 816-825.

[31] Brown CW, Lynch PF, Obremski RJ and Lavery DS. Matrix Representations and Criteria for Selecting Analytical Wavelengths for Multicomponent Spectroscopic Analysis Anal. Chem. 1982; 54: 1472-1479. 